\title{
Progressive Secondary Neurodegeneration and Microcalcification Co-Occur in Osteopontin-Deficient
} Mice

\author{
Walter Maetzler, ${ }^{*}$ Daniela Berg, ${ }^{*}$ Claudia Funke, ${ }^{\dagger}$ \\ Freya Sandmann, ${ }^{*}$ Holger Stünitz, ${ }^{\neq}$ \\ Corina Maetzler, ${ }^{*}$ and Cordula Nitsch ${ }^{\S}$ \\ From the Department of Neurodegenerative Diseases, * Hertie \\ Institute for Clinical Brain Research, German Center for \\ Neurodegenerative Diseases, and the Department of Medical \\ Genetics, ${ }^{\dagger}$ Institute of Human Genetics, University of Tuebingen, \\ Tuebingen, Germany; the Institutt for Geologi, University of Tromsø, \\ Tromsø, Norway; and the Department of Biomedicine, ${ }^{\S}$ Section of \\ Functional Neuroanatomy, University of Basel, Basel, Switzerland
}

In the brain, osteopontin (OPN) may function in a variety of pathological conditions, including neurodegeneration, microcalcification, and inflammation. In this study, we addressed the role of OPN in primary and secondary neurodegeneration, microcalcification, and inflammation after an excitotoxic lesion by examining OPN knock-out (KO) mice. Two, four, and ten weeks after injection of the glutamate analogue ibotenate into the corticostriatal boundary, the brains of 12 mice per survival time and strain were evaluated. OPN was detectable in neuron-shaped cells, in microglia, and at the surface of dense calcium deposits. At this primary lesion site, although the glial reaction was attenuated in OPN-KO mice, lesion size and presence of microcalcification were comparable between OPN-KO and wild-type mice. In contrast, secondary neurodegeneration at the thalamus was more prominent in OPN-KO mice, and this difference increased over time. This was paralleled by a dramatic rise in the regional extent of dense microcalcification. Despite these differences, the numbers of glial cells did not significantly differ between the two strains. This study demonstrates for the first time a genetic model with co-occurrence of neurodegeneration and microcalcification, mediated by the lack of OPN, and suggests a basic involvement of OPN action in these conditions. In the case of secondary retrograde or transneuronal degeneration, OPN may have a protective role as intracellular actor. (Am J Pathol 2010, 177:829-839; DOI: 10.2353/ajpath.2010.090798)
Co-occurrence of neurodegeneration, parenchymal (micro-) calcification, and inflammation can be observed in a number of brain diseases, including Fahr's, Alzheimer's, diffuse Lewy body and Parkinson's disease, Down's syndrome, and hypoxia. ${ }^{1-6}$

Osteopontin (OPN) is a glycophosphoprotein with intra- and extracellular functions influencing cell survival, inflammation, microcalcification and the maintenance of tissue integrity after an injury. ${ }^{7}$ The manifold higher abundance of OPN in cerebrospinal fluid than in blood b,9 $^{8,9}$ argues for a crucial role of this protein in central nervous system (CNS) physiology and pathology. In the developing and adult (rodent) brain, neurons of the olfactory bulb, retina, striatum, and brainstem are OPN-positive. ${ }^{10-13}$ In the aging human brain, OPN is found in pyramidal neurons-more pronounced in Alzheimer's disease ${ }^{14}$-and in dopaminergic neurons of Parkinson's disease patients. ${ }^{8}$ Transient expression of neuronal OPN has been observed under experimental conditions like cryolesioning $^{15}$ and status epilepticus. ${ }^{16}$ In addition, OPN is detectable in microglial cells of lesioned CNS tissue after ischemia, ${ }^{17}$ excitotoxicity, ${ }^{12}$ spinal cord contusion, ${ }^{18}$ as well as in multiple sclerosis plaques ${ }^{19}$ and in microglial cells of the substantia nigra of Parkinson's disease patients. ${ }^{8}$ OPN may also be located extracellularly. ${ }^{8,17}$ The role of OPN in CNS diseases remains controversial. OPN has been shown to be protective in models of stroke ${ }^{17,20,21}$ and spinal cord contusion. ${ }^{18}$ However, OPN inhibited axonal regeneration after injury in the optic nerve, ${ }^{22}$ and the absence of the protein led to a better outcome in models of multiple sclerosis ${ }^{19}$ and Parkinson's disease. ${ }^{8}$

OPN inhibits calcification in bone and at ectopic sites. $^{23-27}$ To our knowledge, the role of OPN in brain microcalcification is unknown. In addition, the co-occurrence of neurodegeneration and microcalcification has

\footnotetext{
Accepted for publication March 31, 2010.

Supplemental material for this article can be found on http://ajp. amjpathol.org.

Address reprint requests to Walter Maetzler, M.D., Hertie Institute for Clinical Brain Research, Otfried-Mueller Strasse 27, 72076 Tuebingen, Germany. E-mail: walter.maetzler@uni-tuebingen.de.
} 
not yet been investigated with a genetic model. In our present study, we were interested in whether OPN deficiency-induced neurodegeneration is paralleled by microcalcification, and which functions of the protein may be primarily involved.

\section{Materials and Methods}

\section{Animals}

OPN-KO mice generated on a Black-Swiss background were backcrossed to C57BI/6 over ten generations, and genotyping was performed as previously described ${ }^{28}$ via PCR of tail biopsies. OPN immunohistochemistry with the polyclonal antibody LF123, a generous gift of Larry Fisher, National Institutes of Health (Bethesda, MD), was performed to confirm the PCR results. C57BL/6J mice were used as wild-type. Ninety-six mice underwent surgical treatment (ie, 12 mice per strain and survival time; two, four, and ten weeks, respectively) received ibotenate, and four mice per strain and survival time served as sham animals. At the day of surgical treatment, the median age of the OPN-KO mice was 127 days, and that of the wild-type mice was 110 days, without significant difference within the compared groups. Weight and sex also did not differ significantly between the groups. All animal experiments were performed with permission of the local animal care committee and in accordance with international guidelines on handling laboratory animals and the present Swiss law.

\section{Excitotoxic Corticostriatal Lesion}

Under combined treatment with atropin (Atropin, 0.05 $\mathrm{mg} / \mathrm{kg} \mathrm{sc}$ ) and burprenorphin (Temgesic, $0.1 \mathrm{mg} / \mathrm{kg} \mathrm{sc}$ ), followed by climazolam (Climasol, $5 \mathrm{mg} / \mathrm{kg} \mathrm{ip}$ ) and ketaminum (Ketamin, $80 \mathrm{mg} / \mathrm{kg} \mathrm{ip}$ ), mice were placed in a stereotaxic apparatus and intracerebral injections performed as detailed previously. ${ }^{29}$ In brief, ibotenate (Sigma, St. Louis, MO; $0.4 \mu \mathrm{g}$ in $0.4 \mu$ l buffered saline) or $0.4 \mu \mathrm{l}$ buffered saline (sham animals) were injected with a Hamilton microsyringe at the coordinates AP $+4.8 \mathrm{~mm}$, $\mathrm{L} 2.1 \mathrm{~mm}$ and $\mathrm{V} 2.5 \mathrm{~mm}$ above ear zero plane according to the atlas of Franklin and Paxinos over a time period of 10 minutes. After further 10 to 15 minutes the needle was withdrawn and the wound closed. To minimize treatment bias, surgery of an OPN-KO mouse was followed by surgery of a wild-type mouse, and such pairs had identical survival times.

\section{Tissue Preparation and Immunohistochemistry}

After recovery periods of two, four, and ten weeks, animals were anesthetized with pentobarbital (Vetanarcol, $0.04 \mathrm{~g} / \mathrm{kg} \mathrm{ip)} \mathrm{and} \mathrm{perfused} \mathrm{with} \mathrm{4 \%} \mathrm{paraformaldehyde}$ and $0.2 \%$ glutaraldehyde in $0.1 \mathrm{~mol} / \mathrm{L}$ phosphate buffer. The brains were then dissected and immersed in $4 \%$ paraformaldehyde overnight. Serial sagittal 40- $\mu \mathrm{m}$ sections were cut with a vibratome and collected in cold TBS. Histology and immunohistochemistry were performed ac- cording to standard protocols, as described previously. ${ }^{29}$ In brief, extent of nerve cell death and position of the needle was determined at the primary lesion site in Cresyl-stained sections (0.5\% Cresyl violet). To evaluate number of micro- and astroglial cells and OPN distribution, iba1, GFAP and OPN stainings were performed with rabbit polyclonal antibodies (anti-iba1, Wako Chemicals, 1:100; anti-GFAP, Sigma, 1:1000; LF123, 1:100). OPN staining was also routinely performed in OPN-KO mice. Immunoreactions were visualized with $0.05 \%$ diaminobenzidine and $0.1 \% \mathrm{H}_{2} \mathrm{O}_{2}$ in TBS. Microcalcification was determined on 1\% Alizarin Red S-stained sections. All sections were mounted on coated slides, dehydrated in graded ethanol, cleared with xylol, and coverslipped in Eukitt (Kindler, Freiburg, Germany).

For visualizing OPN location related to glial cells, double immunofluorescence staining was performed in wild-type mice with a monoclonal anti-OPN antibody (2A1, $0.2 \mu \mathrm{g} / \mathrm{ml}$, raised in OPN-KO mice against recombinant mouse OPN, 1:500) in combination with either the anti-iba1 (1:100), the anti-GFAP antibody (1:1000), or the oligodendrocyte marker (rabbit IgG anti-carbonic anhydrase II from Abcam, 1:200). Alexa 488-conjugated goat anti-mouse and Alexa 568-conjugated goat anti-rabbit (DakoCytomation) were used as secondary antibodies. Colocalization of OPN and calcified deposits was tested with slices double-stained with Alizarin Red S and LF123. Colocalization of OPN and cellular structures was tested with slices doublestained with Cresyl violet and LF123. OPN abundance in the corticostriatal lesion was not only determined in mice with the mentioned survival times but also in mice with four days survival time. Stainings were visualized by an Axiovert 200M Confocal and an Axioplan Microscope (Zeiss, Darmstadt, Germany).

\section{Transmission Electron Microscopy}

Unstained vibratome sections in which presence of calcium deposits was suggested by polarization microscopy or sections neighboring Alizarin Red S-positive serial sections were processed for ultrastructural analysis. ${ }^{29}$ In short, relevant areas were dissected under the microscope and treated for 1 hour with $0.1 \% \mathrm{OsO}_{4}$ or with $1 \% \mathrm{OsO}_{4}$ and $2.5 \% \mathrm{~K}_{2} \mathrm{Cr}_{2} \mathrm{O}_{7}$ to visualize calcium deposition. ${ }^{30}$ Tissue was then dehydrated until $70 \%$ ethanol, blockstained in 1\% uranyl acetate in $70 \%$ ethanol, completely dehydrated with graded ethanol and propylene oxide, and flat-embedded in a plastic resin (Epon; Serva). Ultrathin sections were placed onto copper grids and contrasted with $1 \%$ lead citrate. A CM10 electron microscope (Philips) at $80 \mathrm{kV}$ served for examining the tissue.

\section{Scanning Electron Microscopy}

Vibratome sections from both wild-type and OPN-KO strains with four and ten weeks survival time were placed onto glycerin-coated slides, dehydrated in graded ethanol and air-dried, carbon-coated, and finally sputtered 
with carbon or silver. A Philips CM 30 environmental scanning electron microscope was used for visualization in either the scanning or the back-scatter mode. Elemental analysis was performed with the EDAX EDS-system.

\section{Morphological Analyses}

The size of the corticostriatal neuron-depleted area was determined by circumscribing and analyzing the largest lesion in the cresyl-stained sections (laterality $=2.00$ to $2.20 \mathrm{~mm}$ ) on the screen with the Leica Image Scale system.

The total number of calcium deposits in the corticostriatal lesion was counted on the Alizarin Red S-stained section with the largest lesion (laterality $L=1.95$ to 2.25 $\mathrm{mm})$. According to a previous study, ${ }^{31}$ calcium deposits were further classified into type 1 (transparent deposits often reminiscent of neurons), type 2 (dense core and transparent halo), and type 3 deposits (clearly demarcated dense deposits without halo). This classification may reflect formation steps of the deposits. ${ }^{31}$ In the thalamus, the total area of microcalcification was evaluated using all Alizarin Red S-stained sections exhibiting the thalamic area.

\section{Morphometric Analyses}

Cell number of neurons, identified by the typical morphology of their nuclei in Cresyl-stained sections, of microglia and of astrocytes were estimated according to stereological principles with an optical fractionator, ${ }^{32}$ with systematic random sampling of about every fifth section to yield 3-5 sections/mouse/striatum and 3-4 sections/ mouse/thalamus; for details see. ${ }^{8}$ Stereological analysis was done with a motorized $x-y-z$ stage coupled to a video-microscopy system and the StereologerTM software (Systems Planning and Analysis, Inc., Alexandria, VA). Postprocessing section thickness was measured at each disector location using a focus drive accurate to \pm $0.1 \mathrm{~mm}$ (Applied Scientific Instrumentation, Eugene, OR). The Franklin and Paxinos mouse brain atlas was used for region definitions, and boundaries were identified on each section according to reliable anatomical borders or to the boundary of the lesion. While focusing through relatively thick tissue sections $(18-25 \mu \mathrm{m})$, the top of the nucleus of Cresyl-positive neurons, GFAP-positive astrocytes, or iba1-positive microglia were each identified within disector counting frames $(100 \times 100 \mu \mathrm{m})$. The total number was then computed by multiplying the counted number by the reciprocal of the fraction of the entire striatum/the entire thalamus in which counting was performed (12\% for neurons, microglia and astrocytes, respectively). ${ }^{32}$ All analyses were performed blinded to treatment groups and genotype. Comparison of the morphometric results between the sham animals (OPN-KO and wild-type) did not show significant differences in neither survival time (two, four, and ten weeks). Thus, all eight sham animals per survival time served as control group for calculating the percentage of change of cell numbers in the respective ibotenate-treated animals.

\section{Data Analysis}

Data were analyzed with JMP software (version 7, SAS). Size of the zone of corticostriatal neuron depletion and of dense thalamic microcalcification, as well as morphometric results (numbers of neurons in the thalamus, numbers of micro- and astroglial cells in the thalamus and the corticostriatal lesion) were calculated with the median and range, and significance was determined with the Wilcoxon rank test. Post hoc analyses were performed with analysis of variance. Categorial data were calculated with Fisher's exact test. Areas of microcalcification and microglial abundance in the thalamus were compared using Spearman's Rho. Two-sided $P$ values below 0.05 were considered significant.

\section{Results}

The local neurotoxin injection, which was targeted in the frontal center of the striatum at $L=2.1$ (ie, between the second and third section depicted in Figure 1), resulted in neurodegeneration situated concentrically around the needle tip with a diameter of about $2 \mathrm{~mm}$. There, it did not respect anatomical boundaries but spread via the white matter of the callosal body into the frontal cortex. Next to this primary (corticostriatal) lesion, but spatially clearly separated from it, a secondary neurodegeneration arose which was restricted to a defined brain area, the thalamus. Medial, intermediate, and lateral parts of the thalamus were affected, but some subnuclei of the thalamus were definitely spared (Figure 1; also see below). A detailed analysis of the subnuclei was, however, not the topic of this study.

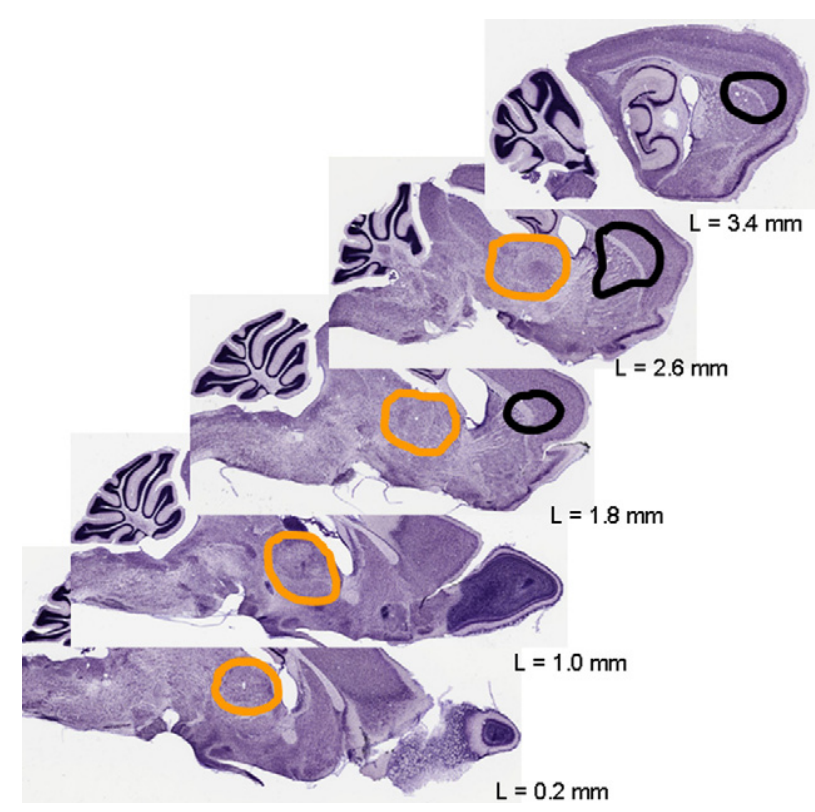

Figure 1. Simulation of the localization and extent of lesions after the excitotoxic insult. The primary excitotoxic lesion includes the striatum and fronto-lateral parts of the cortex (borders marked in black). The zone of secondary neurodegeneration in the thalamus is encircled in orange. Adapted to Nissl-stained sections from BrainMaps.org. L indicates laterality. 


\section{Corticostriatal Lesion}

\section{Qualitative Aspects}

In the corticostriatal region of both strains, ibotenate application induced an area of complete nerve cell loss. A small necrotic center at the site where the capillary tip had been positioned was often observable. The boundary to the healthy tissue was clearly delineated (Figure 2A). This was comparable to previous studies. ${ }^{29,33} \mathrm{Neu}-$ rodegeneration was accompanied by a local increase of microglial (Figure 2B) and astroglial cells (Figure 2C) after two and four weeks, returning to obviously normal density ten weeks after the insult. Calcium deposits in the neuron-depleted area were observed in most of the ibotenate-treated animals, irrespective of whether they were OPN-deficient or wild-type (Figure 2D). The total number of calcium deposits did virtually not differ between the two strains, and, as previously described, type 1 deposits (reminding of neurons, Figure 2E) were generally more abundant after two weeks survival time, and type 3 (Fig-
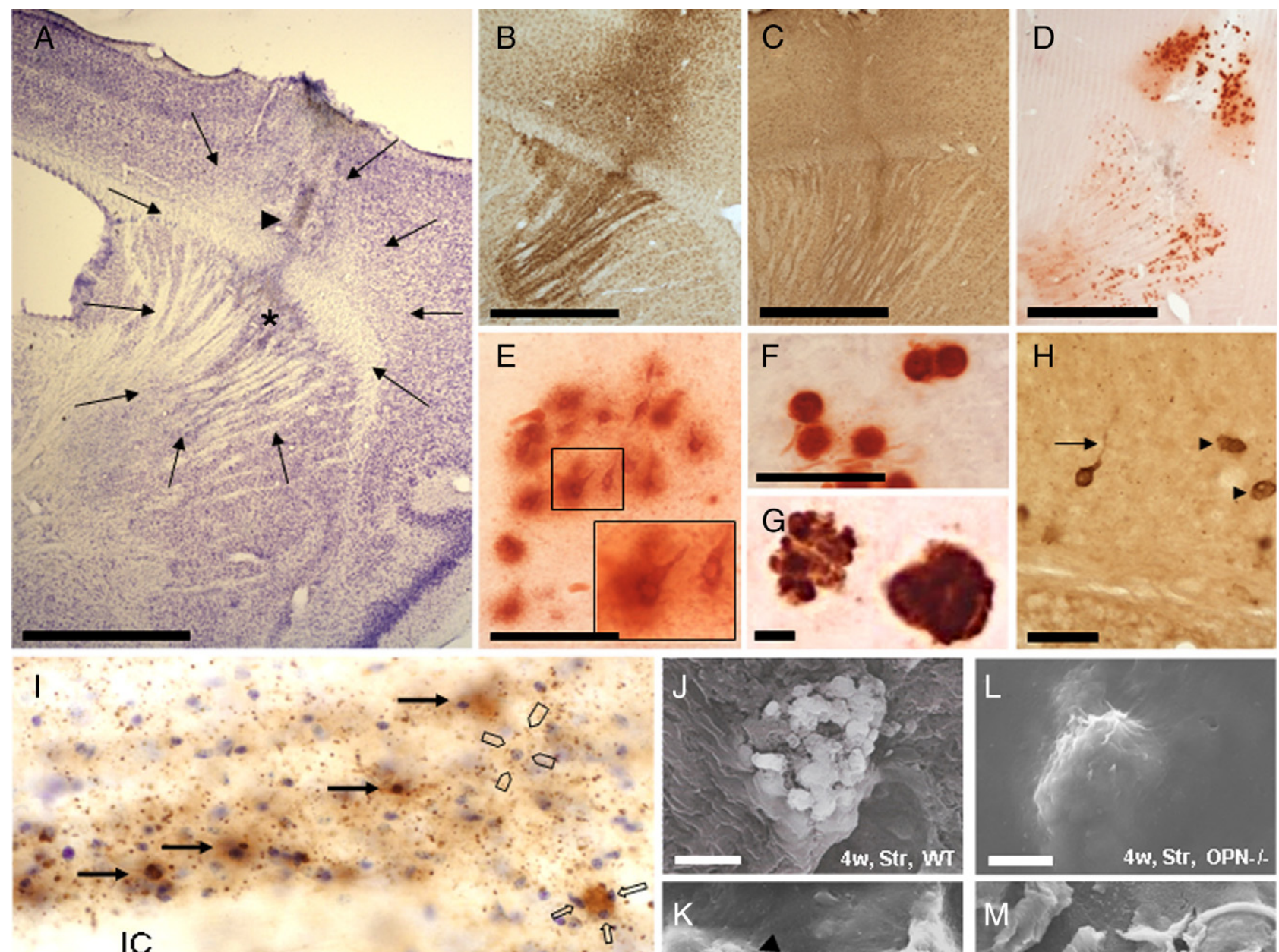

IC
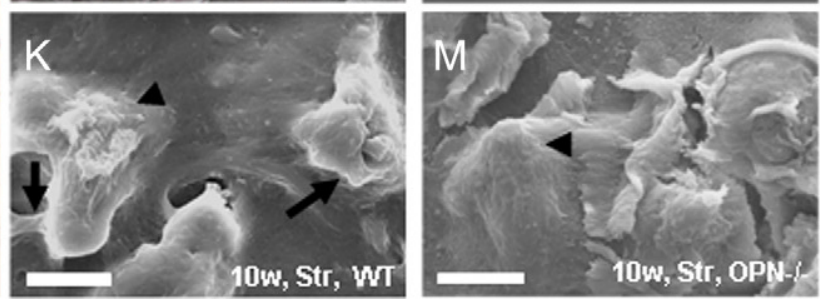

Figure 2. Visual aspects of the corticostriatal lesion. A: The arrows indicate the boundaries of a typical corticostriatal neuron-depleted area in a Cresyl-stained section. The arrowhead indicates the needle tract, the asterisk a small region of central necrosis demarcating the position of the needle-tip. Microglial (B) and astroglial reaction (C) were visualized in iba1- and GFAP-stained sections, and microcalcification with Alizarin Red S stain (D). E: Type 1 (nascent) deposits are often neuron-shaped and have prominent halos (see also inset). F: Type 3 (mature) deposits are round, dense, and have almost no halo. G: Double staining with Alizarin Red S (for calcium deposits) and LF123 (for OPN) indicates a dense association of OPN with calcified material. Note the "bulby" surface of the left deposit. H: Osteopontin (OPN)-stained structures without (arrowheads) and with processes reminding of neurons (arrow) represent osteopontin-positive calcium deposits. I: Section counterstained with Cresyl violet depicts presence of OPN in some neurons (long arrows) where it fills rather homogenously the cytoplasm. Most OPN, however, is found in small-dotted fashion in the neuropil but not in the white matter of the internal capsule (IC). Occasionally dots mark the outer surface of large perikarya (open pointers). An OPN-stained extracellular deposit is associated with small cresyl-positive structures representing most probably microglial nuclei (open arrows). J: In SEM, mainly flat and angular bulbs are observed at the surface of a corticostriatal deposit of a wild-type mouse four weeks after the excitotoxic lesion. K: After ten weeks, these bulbs are often interconnected with bridges (arrows), and parts of the surface of the bulbs appear rough (arrowhead). In OPN knockout mice, corticostriatal deposits have flat and smooth surfaces (arrowhead) without obvious difference between four (L) and ten weeks survival time (M). OPN-/- indicates osteopontin-deficient mouse; w, weeks; WT, wild-type mouse. Scale bars: 1 mm $(\mathbf{A}-\mathbf{D}) ; 50 \mu \mathrm{m}(\mathbf{E}, \mathbf{F}, \mathbf{H}, \mathbf{I}) ; 5 \mu \mathrm{m}$ $(\mathbf{G}, \mathbf{J}, \mathbf{K}, \mathbf{L}, \mathbf{M})$. 
ure 2F) after ten weeks survival time. However, wild-type mice displayed more type 1 deposits, and OPN-KO mice more type 3 deposits at all time points investigated. Double staining with Alizarin Red S and LF123 showed that OPN binds to the surface of the deposits (Figure 2G). In wild-type mice, OPN-positive structures with long processes reminded of neurons (Figure $2 \mathrm{H}$ ). At early survival stages, OPN was homogeneously distributed in cells reminding of neurons, and small-dotted OPN immunoreactivity was observable in the neuropil. Large OPN-positive round structures were associated with small cresyl-positive structures nearby resembling most probably microglial nuclei (Figure 2l). The presence of small-dotted OPN in (but also outside of) microglial cells was confirmed with double immunohistochemistry using iba1 and 2A1 antibodies (this was similar in the corticostriatal and the thalamic region, see also supplemental Video at $h t t p: / /$ ajp.amjpathol.org). After ten weeks, OPN was hardly detectable. Astroglial and oligodendroglial cells never colocalized with OPN (not shown).

Surface topography of corticostriatal deposits was studied with scanning electron microscopy (SEM). After four weeks, wild-type mice demonstrated mainly deposits with flat and angular bulbs (Figure 2J). After ten weeks, bridges connected single bulbs, and parts of the surface of the bulbs appeared rough (Figure 2K). Surface topography of corticostriatal deposits in OPN-KO mice was regularly flat and smooth without obvious differences in surface structure between four and ten weeks postinsult survival time (Figure 2, $\mathrm{L}$ and $\mathrm{M}$ ).

\section{Quantification of Neurodegeneration, Gliosis, and Microcalcification}

Two weeks after the excitotoxic insult, OPN-KO mice showed a smaller area of neuron depletion $\left(1.21 \mathrm{~mm}^{2}\right.$, 0.75-1.98; median, range) than wild-type mice (2.02 $\left.\mathrm{mm}^{2}, 1.16-2.47, P=0.002\right)$. Later on, the extent of neurodegeneration did not differ between the strains $\left(1.92 \mathrm{~mm}^{2}, 0.72-2.98\right.$ in OPN-KO mice versus $1.98 \mathrm{~mm}^{2}$, $1.17-3.10$ in wild-type mice after four weeks, $P=0.39$; $1.60 \mathrm{~mm}^{2}, 0.61-2.57$ in OPN-KO mice versus $2.00 \mathrm{~mm}^{2}$, 1.26-2.37 in wild-type mice after ten weeks, $P=0.12$, Figure $3 \mathrm{~A}$ ).

The number of iba1-positive microglial cells multiplied in the vicinity of the ibotenate-induced lesion, but the percentage of increase compared with sham animals was generally lower in OPN-KO mice than in wild-type mice (after two weeks, 242\%, 169-440 in OPN-KO mice versus $384 \%, 287-459$ in wild-type mice, $P=0.02$; after four weeks, $247 \%, 93-451$ versus $427 \%, 118-609, P=$ 0.04; after ten weeks, $164 \%, 102-259$ versus $258 \%$, 99 $420, P=0.12$, Figure 3B). The number of iba1-positive cells dropped in both strains significantly from four weeks to ten weeks survival time.

Similarly, percentages of increases in the number of GFAP-positive cells, compared with sham animals, were generally lower in the corticostriatal lesion of OPN-KO compared with wild-type mice (after two weeks, $148 \%$, $122-233$ in OPN-KO mice versus 193\%, 78-273 in wildtype mice, $P=0.05$; after four weeks, 168\%, 115-205
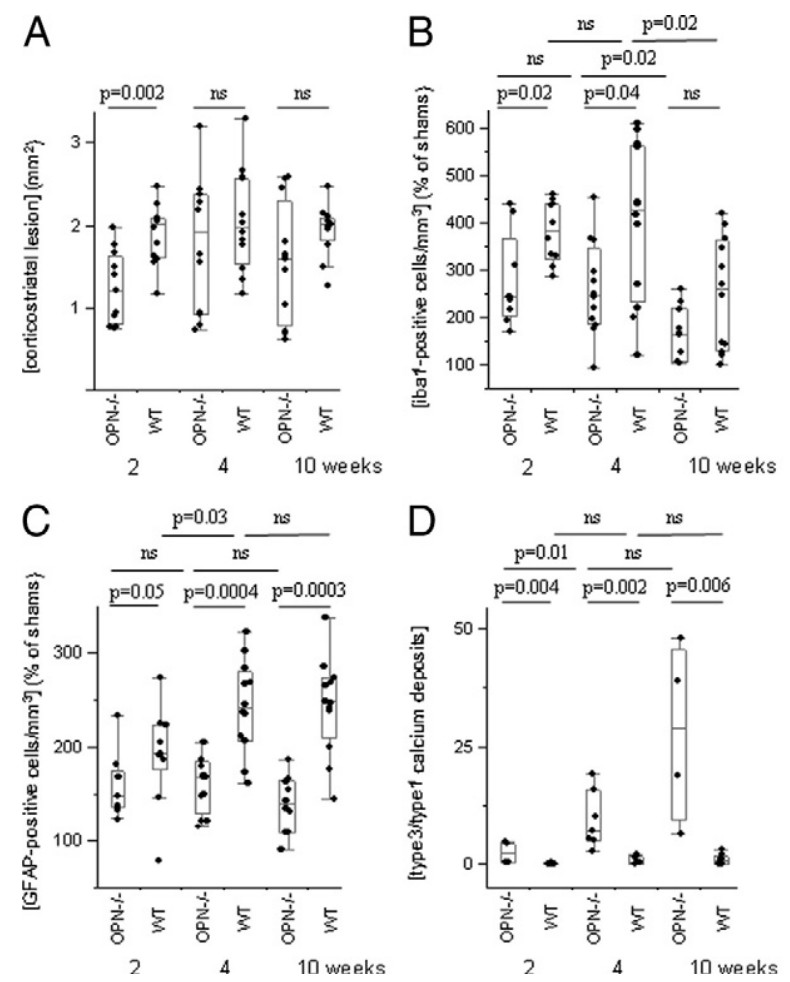

Figure 3. Morphological and morphometric analyses of the corticostriatal lesion. A: The corticostriatal lesion was larger in wild-type than in OPN-KO mice two weeks after the excitotoxic insult but did not differ significantly at later time points. B: Number of microglial cells was higher in wild-type than in OPN-KO mice two and four weeks after lesioning, and returned to lower levels after ten weeks, now without significant difference between the strains. C: GFAP-positive cells were more numerous in wild-type than in OPN-KO mice at all time points investigated. D: The ratio of the deposits (type3/type 1) was higher in OPN-KO than in wild-type mice at all investigated time points.

versus $241 \%, 161-322, P=0.0004$; after ten weeks, $139 \%, 90-185$ versus $247 \%, 144-336, P=0.0003$, Figure 3C). In wild-type mice, GFAP-positive cells were significantly more abundant after four weeks than after two weeks survival time.

The number of calcium deposits did not significantly differ between the strains at the three observation points (not shown). However, the increase of the ratio type 3 (round deposits without halo)/type 1 (neuron-shaped) calcium deposits from two to ten weeks survival time (Figure 3D) indicates an increased mineralization of deposits in OPN-KO mice.

\section{Thalamic Degeneration}

\section{Qualitative Aspects}

A progressive loss of thalamic neurons was obvious in OPN-KO mice after the excitotoxic corticostriatal insult (Figure 4, Cresyl). OPN immunoreactivity in wild-type mice, as assessed with the LF123 antibody, did not differ in its distribution from the situation in the corticostriatal lesion, including presence of homogeneously distributed OPN in cells reminding of neurons, and small-dotted OPN immunoreactivity in the neuropil (see Figure 2, $\mathrm{H}$ and I). After ten weeks, OPN was hardly detectable (Figure 4, 


\section{Cresyl}
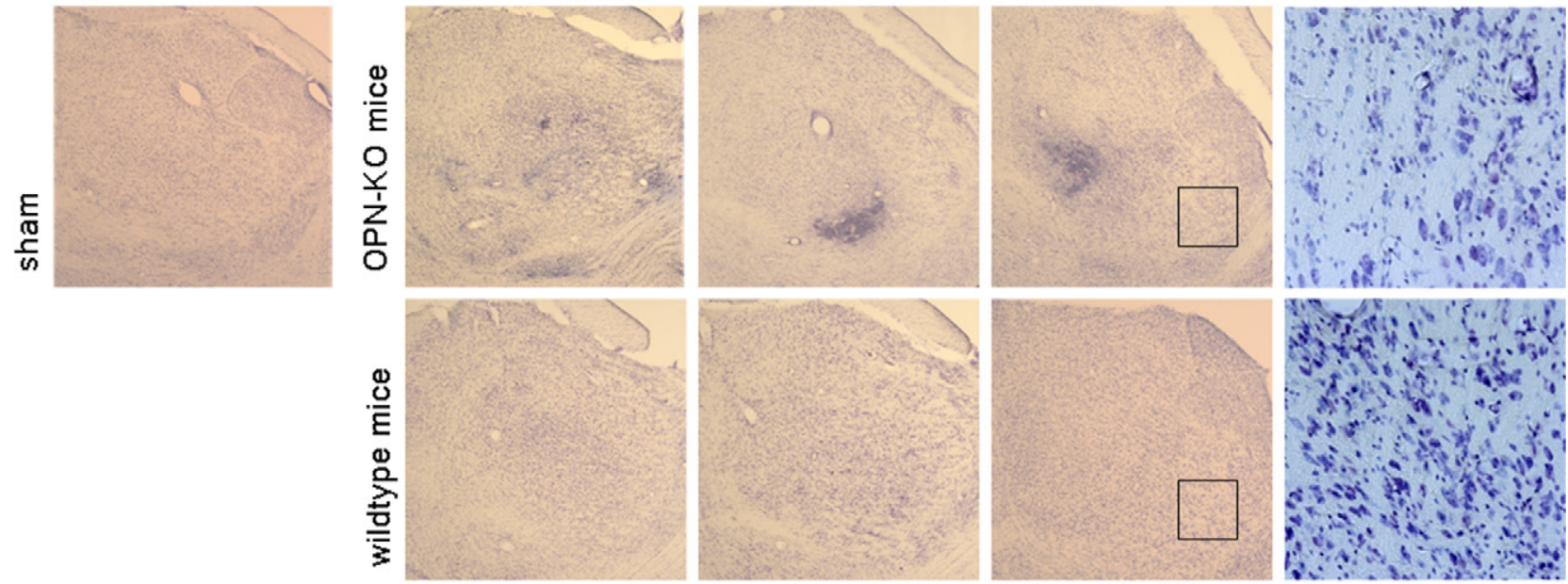

\section{Osteopontin}
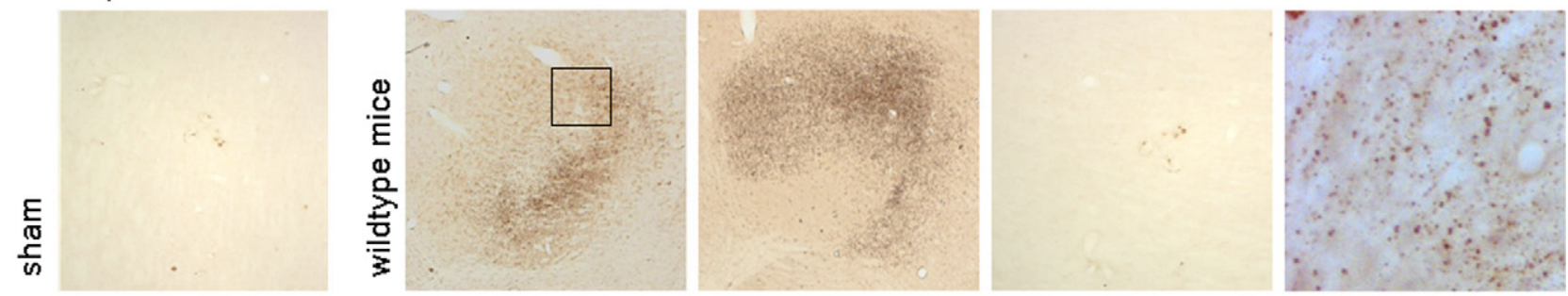

iba1
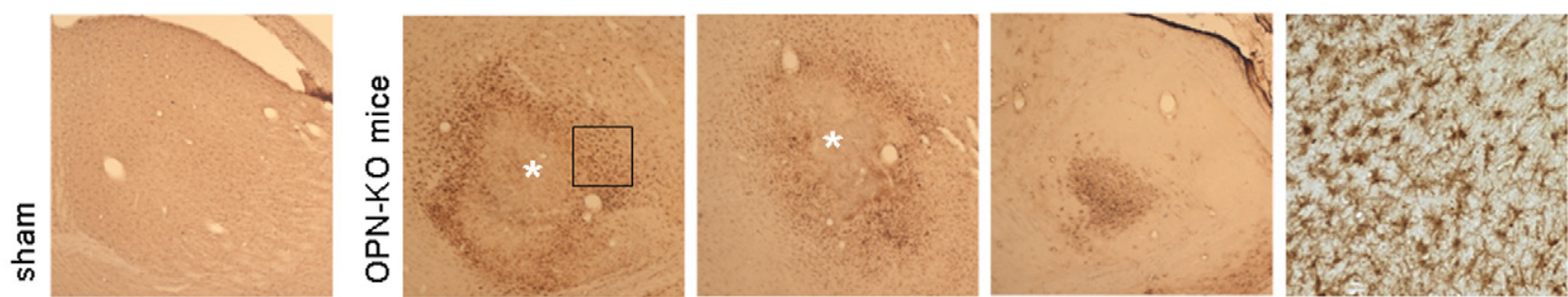

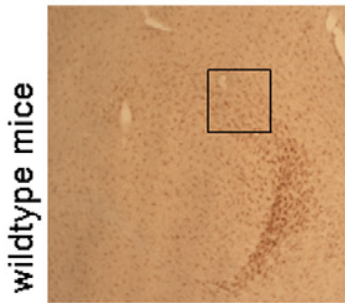

2 weeks

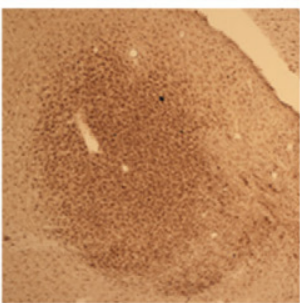

4 weeks

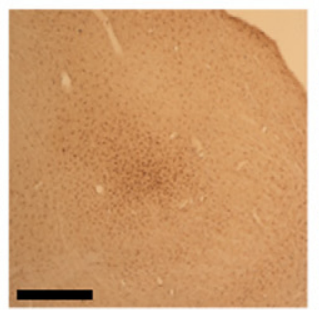

10 weeks

Figure 4. Visual aspects of the thalamic degeneration. Cresyl staining reveals a massive and progressive neuronal loss in the thalamus of OPN-KO mice. Note also the dense structures that coincide with the regions where dense microcalcification occurs. In ibotenate-treated wild-type mice, there is a mild and non-progressive rarification of thalamic neurons observable. Osteopontin: In wild-type mice, OPN-stained sections (LF123 antibody) show an abundance of small dots in thalamic regions two and four weeks after the corticostriatal insult. Iba1-treated sections reveal microglia, which appears more activated in the OPN-KO mice. Note the intrathalamic necrosis in OPN-KO mice marked by the asterisks. Areas indicated by rectangles are shown at higher magnifications at the right side of the figure. Scale bar $=300 \mu \mathrm{m}$.

Osteopontin). Double-immunofluorescence revealed that much but not all small-dotted OPN was located in microglial cells, at the basis of microglial processes (supplemental Video at http://ajp.amjpathol.org). Diffuse OPN staining was occasionally detectable in double immunofluorescence but never colocalized with glia.

lba1 staining revealed upregulation of microglial cells in the affected area in particular after four weeks, with virtually more intense staining in the OPN-KO strain. In OPN-KO mice but not in wild-type mice, intrathalamic necrotic areas were repeatedly observable (Figure 4, iba1). They resemble tissue softening in lacunar microinfarcts and lack microglial cells. A moderate activation of thalamic astroglia, assessed on GFAP-stained sections, was regularly seen after two and four weeks survival time in both strains (not shown). 


\section{Alizarin red S, SEM}
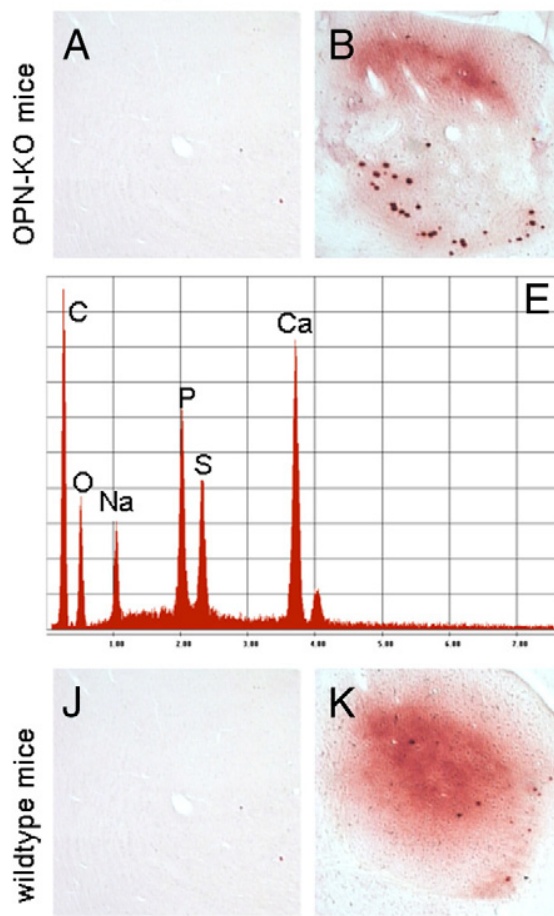

2 weeks
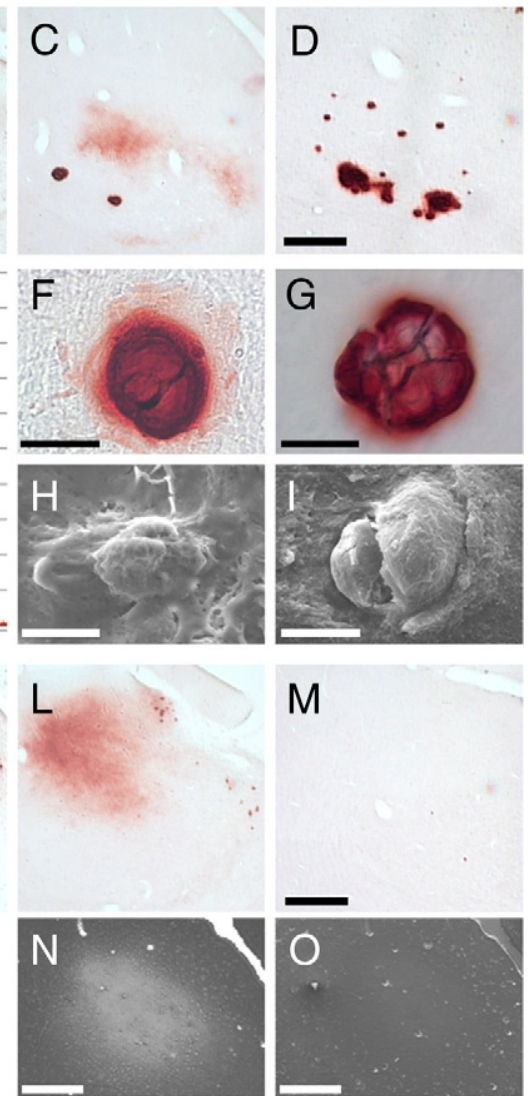

10 weeks
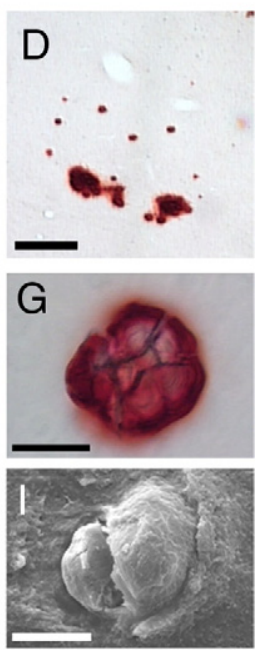

M

4 weeks

Degenerated shrunken presynaptic boutons could occasionally be recognized by their preserved postsynaptic contacts (Figure 6C). More frequent, however, were darkly degenerated "postsynaptic" structures, ie, neuronal perikarya (Figure 6D) and their dendrites (Figure 6E). Neuronal perikarya often evidenced signs of an ongoing pathological process: cytoplasmic fibrillar inclusion bodies (as found in the thalamus in humans and experimental animals in a number of neurodegenerative diseases ${ }^{34,35}$ ) and accumulations of lysosomes were situated in otherwise well preserved neuronal cell bodies (Figure 6F). These features were found in both strains. They suggest that next to anterograde (terminal) degeneration, the corticostriatal lesion caused a transneuronal and/or retrograde degeneration of thalamic/thalamocortical neurons. On the basis of their ultrastructural features, these different forms of distant neurodegeneration cannot be differentiated.

Occasionally, small concretions with ultrastructural characteristics of calcium deposits and not associated with clearly identifiable neuronal or glial compartments were found in both species while large layered deposits $^{36}$ were only detected in OPN-KO mice (not shown).

\section{rably small and presented with a halo.} dense deposits were rare and if present, they were compa-

At the ultrastructural level, degenerated myelinated axons were abundant in the degenerated parts of the thalamus after two weeks survival (Figure 6, A-C). Preserved and damaged axons were often neighboring each other (Figure 6B). Frequently, swollen mitochondria in the degenerated axoplasm stained positive for calcium (Figure 6B).

\section{Quantification of Neurodegeneration, Gliosis, and Microcalcification}

Two weeks after the excitotoxic insult, both strains exhibited a significant loss of thalamic neurons, com- 

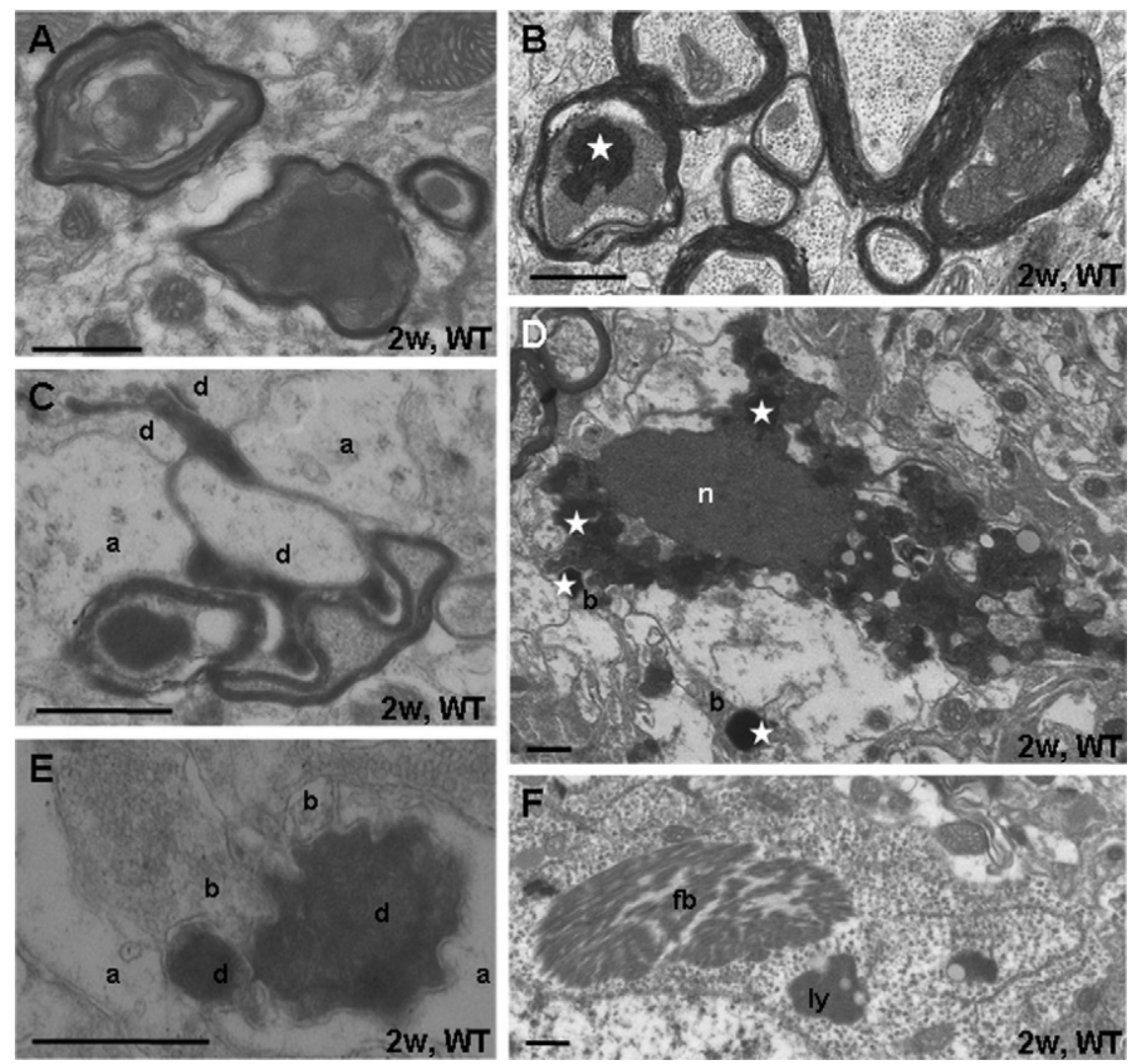

Figure 6. Ultrastructure of the thalamic degeneration. A: Degenerated myelinated axons display swollen mitochondria and darkly degenerated masses. B: Sections treated with potassium chromate show that mitochondria in degenerating axons contain high calcium levels (white asterisk). C: Dark degenerated corticothalamic axon terminals maintain synaptic contact zones with dendrites (d); a, astroglial process. D: Degenerated neuron with dense nucleus (n) and dense cytoplasm contains swollen mitochondria, partly staining for calcium deposition (white asterisk). They are also found in degenerated boutons (b). Section was treated with potassium bichromate. E: Dark degenerated dendrite (d) partially engulfed by astroglia (a) maintains contacts with presynaptic boutons (b). F: Neuronal perikarya contain fibrillar bodies (fb) and accumulations of lysosomes (ly). Scale bar $=0.5 \mu \mathrm{m}$ pared with sham-treated animals $(P<0.0001)$. In addition, OPN-KO mice (61.5\% of sham, 49.5-72.7) had significantly fewer thalamic neurons after two weeks survival time, than wild-type animals $(68.9 \%, 51.8-79.4, P=$ 0.03). Four and ten weeks after the excitotoxic lesion, the difference increased further (after four weeks, OPN-KO mice, 54.3\%, 35.9-70.4; wild-type mice, 66.6\%, 48.190.7, $P=0.003$; after ten weeks, OPN-KO mice, $44.2 \%$, 34.9-56.6; wild-type mice, 65.7\%, 47.4-85.9, $P<$ $0.0001)$. The progressive neuronal loss, ie, the reduction of cells from two to ten weeks survival time, in OPN-KO mice was highly significant $(P=0.0001)$ (Figure 7A). In wild-type mice, there was no significant difference between the different survival times $(P=0.72)$.

In general, the percentage of microglial cells in ibotenate- compared with sham-treated animals was $96 \%$ higher after two, four, and ten weeks observation period $(P<0.0001)$. The highest numbers of microglial cells were observed after four weeks, irrespective of the strain investigated (Figure 7B). At defined survival times, there were no significant differences of microglial abundance between OPN-KO and wild-type mice. In particular, microglial cells reached $163 \%$ of sham (100-218) in OPN-KO, and $125 \%(100-225)$ in wild-type mice two weeks after ibotenate treatment $(P=0.50), 221 \%(177-331)$ in OPN-KO and $248 \%$ (147-307) in wild-type mice after four weeks ( $P=$ $0.47)$, and $194 \%$ (124-232) in OPN-KO and 155\% (125$212)$ in wild-type mice after ten weeks $(P=0.39)$.

Overall, GFAP-positive cells were about $40 \%$ more abundant in ibotenate- compared with sham-treated animals $(P=0.0008)$. However, significant differences were neither detectable between strains at similar time points nor between time points in similar strains (Figure 7C).

Thirty-four of 36 ibotenate-treated OPN-KO mice (11/12 after two weeks, 11/12 after four weeks, 12/12 after ten weeks survival time), and four of 36 wild-type mice (0/12 after two weeks, 3 of 12 after four weeks, $1 / 12$ after ten weeks) developed dense thalamic calcium deposits ( $P=0.0001$, Fisher's exact test). In OPN-KO mice, the area of dense microcalcification increased with longer survival times and was significantly larger than in wildtype mice at all time points investigated (Figure 7D). Areas of microcalcification and microglial abundance correlated in wild-type mice (Rho $=0.45, P=0.026)$ but not in OPN-KO mice (Rho $=0.08, P=0.64)$.

\section{Discussion}

We found OPN located in neuron-shaped structures, in microglial cells, and at calcium deposits after excitotoxicity. This provides evidence that OPN is involved in neurodegeneration, microcalcification, and inflammation. In addition, progressive secondary neurodegeneration co-occurs with microcalcification in OPN-KO but not wildtype mice.

At the primary lesion site where neurodegeneration is caused by the excitotoxic glutamate analogue, the area of nerve cell loss was smaller in OPN-KO mice compared with wild-type mice two weeks after ibotenate injection; this difference was no longer observable at later survival times. In addition, micro- and astrogliosis were signifi- 
A
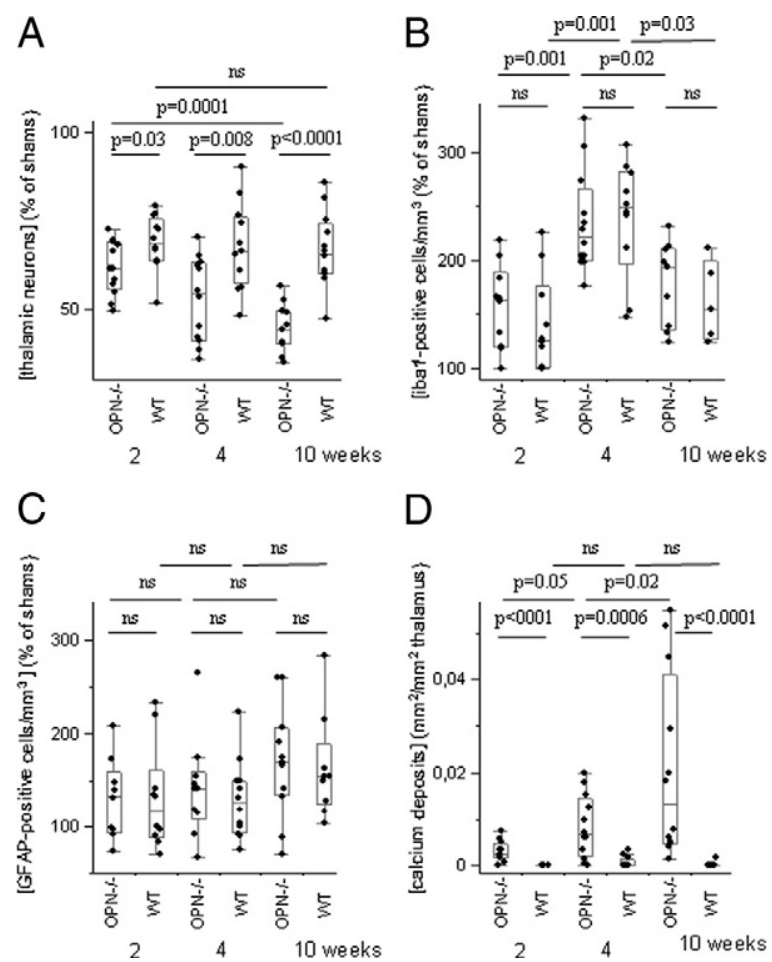

Figure 7. Morphological and morphometric analyses of the thalamus. A OPN-KO mice had fewer thalamic neurons than wild-type mice two, four, and ten weeks after the excitotoxic corticostriatal lesion, this loss being progressive only in OPN-KO mice. B: Numbers of microglial cells did not differ significantly between OPN-KO and wild-type mice, and peaked after four weeks survival. C: GFAP-positive cells were more numerous in ibotenate-treated mice compared with sham animals, but their numbers did not differ significantly between ibotenate-treated strains and survival time. D: The total area of thalamic microcalcification was higher in OPN-KO than in wild-type mice and increased further over time.

cantly attenuated in OPN-KO mice, in particular at "early" investigation stages. These findings are basically in line with a recent study that found an unaltered infarct development at the primary injury site between OPN-KO and wild-type mice, ${ }^{17}$ and with another study that detected lowered mRNA levels of inflammatory proteins in OPN-KO mice after spinal contusion. ${ }^{18}$ Our data suggest that the attenuation of the inflammatory process caused by the lack of OPN may delay the neurodegenerative process after an excitotoxic lesion, but has ultimately no beneficial effect on neuronal survival. This may, at least partly, be explained by the reduced abundance of activated astrocytes in OPN-KO mice as observed in this study and points to a relevant role of OPN in the organization of the glial scare and in preventing the organism from further damage through inadequate glial response.

Thus, the function of OPN at the primary lesion site may more focus on its inflammation-modulating properties, contrary to a possibly more protective role as an intracellular actor at distant lesion sites (see below).

In agreement with our previous study, ${ }^{31}$ we found microcalcification at the primary lesion site. At this location, where neurons survive only for a short time (which, however, may be sufficient for the nucleation of crystals), number of calcified deposits did not differ between the OPN-KO and the wild-type strain. Thus, OPN may not inhibit crystal nucleation relevantly or may be up-regulated too late after the excitotoxic insult to exert this function -in fact, a potent inhibitory effect of hydroxyapatite formation by OPN phosphopeptides has been demonstrated. ${ }^{23}$ Rather, OPN may modulate the subsequent formation of crystals in damaged (but primarily surviving) neurons. ${ }^{24}$ We provide indirect evidence for this hypothesis by showing that i) Alizarin Red S-stained deposits at early stages remind of neurons (Figure 2E), ii) OPN is diffusely distributed in neuron-like structures (Figure 2, H and I), iii) OPN-KO mice have more type 3 deposits at all time points investigated, indicating an increased mineralization of crystal aggregates in OPN-KO mice, and iv) deposits of OPN-KO mice have a flat and smooth surface topography without obvious differences between four and ten weeks postinsult survival time (indicating little mineral-matrix interaction), in contrast to the surface structure of corticostriatal crystals of wild-type mice, which change obviously between four and ten weeks postlesion time, and exert signs of active regulation (in our model reflected by Alizarin Red S-stained deposits with halo, Figure 2). Using a transgenic mouse model with selective neuronal expression of the calcium-binding protein parvalbumin we recently demonstrated that neurons are in fact hosts for crystal nucleation and are therefore crucially involved in ectopic microcalcification. ${ }^{31}$ The rough and "bulby" surface with interconnecting bridges observed in deposits of wild-type mice with longer survival may be best explained by the known region-specific organization of the organic matrix network on crystallographic faces. ${ }^{37}$ Taken together, these data make it improbable that crystal nucleation in the brain occurs (mainly) extracellularly. We hypothesize that, if the intraneuronal growth process of the crystal cannot be adequately modulated/inhibited (eg, by direct adsorption of intraneuronal OPN to the surface of the deposit, but also by other regulatory factors), the deposit will reach a dimension that interferes with cellular integrity, followed by neuronal degeneration, and consecutively by the extracellular location of the crystal. At this stage, the crystal surface topography will be modified by an organic matrix network, which consists of procalcific and anticalcific regulatory factors, now including phagocytes. ${ }^{24,25,38}$ Dependent on the balance between these factors, the crystal will then either be removed, growth will be inhibited, or the crystal will further grow. An incomplete removal will finally result in an (almost) inactive stage of the dystrophic calcification process (in our model reflected by Alizarin Red S-stained deposits without a halo, Figure 2F).

At the secondary lesion site, the thalamus, anterograde "Wallerian" degeneration of axons and their terminals from neuronal perikarya situated in the primary lesion area is an inherent necessity. However, in addition, there was a massive loss of neuronal perikarya in the thalamus, which cannot be explained by a direct exposure to the excitotoxin. Rather, retrograde and/or anterograde transneuronal degeneration ${ }^{39}$ was abundant. This was more prominent in OPN-KO mice two weeks after lesioning and continued in this strain over the ten weeks observation period. Glial reaction to this secondary neurodegeneration was mild-the relative microglial cell 
numbers were up to threefold increased (Figure 7B), compared with a two- to sixfold increase at the primary lesion site (Figure 3B) - and did not differ between the strains (ie, the ongoing secondary degeneration in OPN-KO mice was not accompanied by an increased glial reaction), suggesting that the inflammation-modulating properties of OPN may not be critical for this process. However, with the stereological approach used in this study we cannot definitely exclude that microglial cell numbers were higher than in wild-type mice: $\mathrm{KO}$ mice often showed necrotic areas in the thalamus, which were always free of microglia (see also Figure 4, iba1 staining, asterisks). In addition, to omit selection bias, we counted cells in the whole thalamus and not in subnuclei (eg, the ventrolateral thalamus) because the boundaries of the subnuclei are not clear-cut. This may partly explain the different outcomes between this study and a recent publication. ${ }^{17}$ Nevertheless, the lack of a significant difference between groups of 12 mice, and the unbiased and three-dimensional approach of quantifying cells argue, at least, for further relevant mechanisms involved in secondary neurodegeneration, and ectopic calcification.

The distant thalamic degenerative area exhibited a massive and progressive ectopic calcification process over ten weeks observation period in parallel with the ongoing secondary neurodegeneration. In addition, dense thalamic calcium deposits occurred in eight of 12 shamtreated OPN-KO animals with two or more weeks survival time, but in no sham-treated wild-type animals. These findings argue for a massively reduced capacity of this genetically altered system to counteract the nucleation and subsequent formation of calcium aggregates.

Assuming that neurons are the (primary) source for calcium deposits ${ }^{31,36,40}$ (see also above) it is reasonable to suggest that neurons that have intracellular nucleation of calcium aggregates may express OPN to keep crystal size to a minimum and finally to dissolve these aggregates by the physical presence and activity of OPN. Similar to the findings in the corticostriatal lesion, we found neuron-shaped structures in the affected thalamus, which stained diffusely for OPN, indicating intraneuronal OPN abundance, but also small-dotted OPN immunoreactivity in and outside of microglial cells (supplemental Video at $h$ ttp://ajp.amjpathol.org). At a light microscopic level, OPN was abundant where misty Alizarin Red S staining was observable, and formation of concrete deposits was rare (Figure 4, osteopontin, and Figure 5, $\mathrm{K}-\mathrm{M})$. We therefore hypothesize that OPN inhibits nucleation as well as subsequent formation of mineral deposits not only in peripheral tissues ${ }^{26,41}$ or in in vitro models, ${ }^{23,42}$ but also in vivo in the central nervous system. However, this needs to be proven in subsequent studies.

It also remains to be proven whether the inflammatory response in the thalamic area is (not) crucially involved in this calcification process. The dramatic difference of thalamic mineralization between OPN-KO and wild-type mice without significant differences in microglial cell numbers, and the relatively mild inflammation in the thalamus compared with the primary lesion site (where, vice versa, no significant quantitative differences in microcalcification could be observed) point to a minor role of these cells in the microcalcification process at the secondary lesion site. This is in line with previous studies demonstrating a relevant inhibitory role of endogenously produced OPN on parenchymal cell calcification, which occurs in the absence of inflammatory cells. ${ }^{42}$ However, the occurrence of thalamic calcium deposits in three of our wild-type animals after four weeks survival time but only in one after ten weeks survival time, and the positive correlation of microcalcification and microglial abundance in the thalamus of wild-type mice (but not OPN-KO mice) argue for an involvement of inflammation and phagocytosis at least in the subsequent formation and removal of crystals.

In conclusion, with these results we provide evidence that the lack of OPN induces co-occurring progressive secondary neurodegeneration and microcalcification. Extracellular functions of OPN like modulation of inflammation may not be primarily involved.

\section{Acknowledgments}

We thank Lucy Liaw for generously providing the OPN mouse strain, Larry Fisher (National Institutes of Health) for providing the LF123 antibody, and Susan Rittling (The Forsyth Institute, Boston, Massachusetts) for providing the 2A1 antibody. The excellent technical help of Olga Bollag and Gabriela Kalt is greatly appreciated.

\section{References}

1. Nagaratnam N, Plew JD: Extensive intracranial calcification secondary to hypoxia, presenting with dyspraxic gait. Australas Radiol 1998, 42:232-233

2. Rodriguez MJ, Ursu G, Bernal F, Cusi V, Mahy N: Perinatal human hypoxia-ischemia vulnerability correlates with brain calcification. Neurobiol Dis 2001, 8:59-68

3. Bouras C, Giannakopoulos P, Good PF, Hsu A, Hof PR, Perl DP: A laser microprobe mass analysis of trace elements in brain mineralizations and capillaries in Fahr's disease. Acta Neuropathol (Berl) 1996, 92:351-357

4. Mann DM: Calcification of the basal ganglia in Down's syndrome and Alzheimer's disease. Acta Neuropathol (Berl) 1988, 76:595-598

5. Ramonet D, de Yebra L, Fredriksson K, Bernal F, Ribalta T, Mahy N: Similar calcification process in acute and chronic human brain pathologies. J Neurosci Res 2006, 83:147-156

6. Vermersch P, Leys D, Pruvo JP, Clarisse J, Petit H: Parkinson's disease and basal ganglia calcifications: prevalence and clinicoradiological correlations. Clin Neurol Neurosurg 1992, 94:213-217

7. Scatena M, Liaw L, Giachelli CM: Osteopontin: a multifunctional molecule regulating chronic inflammation and vascular disease. Arterioscler Thromb Vasc Biol 2007, 27:2302-2309

8. Maetzler W, Berg D, Schalamberidze N, Melms A, Schott K, Mueller JC, Liaw L, Gasser T, Nitsch C: Osteopontin is elevated in Parkinson's disease and its absence leads to reduced neurodegeneration in the MPTP model. Neurobiol Dis 2007, 25:473-482

9. Maetzler W, Michelis J, Tomiuk J, Melms A, Becker C, Gasser T, Schulte C, Berg D: A single-nucleotide polymorphism of the osteopontin gene may contribute to a susceptibility to Lewy body disease. J Neural Transm 2009, 116:599-605

10. Lee MY, Choi JS, Lim SW, Cha JH, Chun MH, Chung JW: Expression of osteopontin mRNA in developing rat brainstem and cerebellum. Cell Tissue Res 2001, 306:179-185

11. Shin SL, Cha JH, Chun MH, Chung JW, Lee MY: Expression of osteopontin mRNA in the adult rat brain. Neurosci Lett 1999, 273:73-76

12. Chidlow G, Wood JP, Manavis J, Osborne NN, Casson RJ: Expres- 
sion of osteopontin in the rat retina: effects of excitotoxic and ischemic injuries. Invest Ophthalmol Vis Sci 2008, 49:762-771

13. Iczkiewicz J, Rose S, Jenner P: Osteopontin (Eta-1) is present in the rat basal ganglia. Brain Res Mol Brain Res 2004, 132:64-72

14. Wung JK, Perry G, Kowalski A, Harris PL, Bishop GM, Trivedi MA, Johnson SC, Smith MA, Denhardt DT, Atwood CS: Increased expression of the remodeling- and tumorigenic-associated factor osteopontin in pyramidal neurons of the Alzheimer's disease brain. Curr Alzheimer Res 2007, 4:67-72

15. Shin T, Ahn M, Kim H, Moon C, Kang TY, Lee JM, Sim KB, Hyun JW: Temporal expression of osteopontin and CD44 in rat brains with experimental cryolesions. Brain Res 2005, 1041:95-101

16. Borges K, Gearing M, Rittling S, Sorensen ES, Kotloski R, Denhardt DT, Dingledine R: Characterization of osteopontin expression and function after status epilepticus. Epilepsia 2008, 49:1675-1685

17. Schroeter M, Zickler P, Denhardt DT, Hartung HP, Jander S: Increased thalamic neurodegeneration following ischaemic cortical stroke in osteopontin-deficient mice. Brain 2006, 129:1426-1437

18. Hashimoto M, Sun D, Rittling SR, Denhardt DT, Young W: Osteopontin-deficient mice exhibit less inflammation, greater tissue damage, and impaired locomotor recovery from spinal cord injury compared with wild-type controls. J Neurosci 2007, 27:3603-3611

19. Chabas D, Baranzini SE, Mitchell D, Bernard CC, Rittling SR, Denhardt DT, Sobel RA, Lock C, Karpuj M, Pedotti R, Heller R, Oksenberg JR, Steinman L: The influence of the proinflammatory cytokine, osteopontin, on autoimmune demyelinating disease. Science 2001, 294:1731-1735

20. Doyle KP, Yang T, Lessov NS, Ciesielski TM, Stevens SL, Simon RP, King JS, Stenzel-Poore MP: Nasal administration of osteopontin peptide mimetics confers neuroprotection in stroke. J Cereb Blood Flow Metab 2008, 28:1235-1248

21. Meller R, Stevens SL, Minami M, Cameron JA, King S, Rosenzweig H, Doyle K, Lessov NS, Simon RP, Stenzel-Poore MP: Neuroprotection by osteopontin in stroke. J Cereb Blood Flow Metab 2005, 25:217-225

22. Kury P, Zickler P, Stoll G, Hartung HP, Jander S: Osteopontin, a macrophage-derived matricellular glycoprotein, inhibits axon outgrowth. FASEB J 2005, 19:398-400

23. Pampena DA, Robertson KA, Litvinova O, Lajoie G, Goldberg HA, Hunter GK: Inhibition of hydroxyapatite formation by osteopontin phosphopeptides. Biochem J 2004, 378:1083-1087

24. Jahnen-Dechent W, Schafer C, Ketteler M, McKee MD: Minera chaperones: a role for fetuin-A and osteopontin in the inhibition and regression of pathologic calcification. J Mol Med 2008, 86:379-389

25. Giachelli CM: Inducers and inhibitors of biomineralization: lessons from pathological calcification. Orthod Craniofac Res 2005, 8:229-231

26. Speer MY, McKee MD, Guldberg RE, Liaw L, Yang HY, Tung E, Karsenty G, Giachelli CM: Inactivation of the osteopontin gene enhances vascular calcification of matrix gla protein-deficient mice: evidence for osteopontin as an inducible inhibitor of vascular calcification in vivo. J Exper Med 2002, 196:1047-1055

27. Steitz SA, Speer MY, McKee MD, Liaw L, Almeida M, Yang H, Giachelli CM: Osteopontin inhibits mineral deposition and promotes regression of ectopic calcification. Am J Pathol 2002, 161:2035-2046

28. Liaw L, Birk DE, Ballas CB, Whitsitt JS, Davidson JM, Hogan BL: Altered wound healing in mice lacking a functional osteopontin gene (spp1). J Clin Invest 1998, 101:1468-1478

29. Maetzler W, Nitsch C, Bendfeldt K, Racay P, Vollenweider F, Schwaller B: Ectopic parvalbumin expression in mouse forebrain neurons increases excitotoxic injury provoked by ibotenic acid injection into the striatum. Exp Neurol 2004, 186:78-88

30. Probst W: Ultrastructural localization of calcium in the CNS of vertebrates. Histochemistry 1986, 85:231-239

31. Maetzler W, Stunitz H, Bendfeldt K, Vollenweider F, Schwaller B, Nitsch C: Microcalcification after excitotoxicity is enhanced in transgenic mice expressing parvalbumin in all neurones, may commence in neuronal mitochondria and undergoes structural modifications over time. Neuropathol Appl Neurobiol 2009, 35:165-177

32. West MJ: New stereological methods for counting neurons. Neurobiol Aging 1993, 14:275-285

33. Schwarcz R, Hokfelt T, Fuxe K, Jonsson G, Goldstein M, Terenius L: Ibotenic acid-induced neuronal degeneration: a morphological and neurochemical study. Exp Brain Res 1979, 37:199-216

34. Sato H, Sato M: Ultrastructural morphology of thalamic cytoplasmic inclusion bodies in El mouse. Exp Neurol 1986, 93:160-167

35. Wisniewski HM, Berry K, Spiro AJ: Ultrastructure of thalamic neuronal inclusions in myotonic dystrophy. J Neurol Sci 1975, 24:321-329

36. Nitsch C, Scotti AL: Ibotenic acid-induced calcium deposits in rat substantia nigra. Ultrastructure of their time-dependent formation, Acta Neuropathol (Berl) 1992, 85:55-70

37. Chien YC, Hincke MT, Vali H, McKee MD: Ultrastructural matrixmineral relationships in avian eggshell, and effects of osteopontin on calcite growth in vitro. J Struct Biol 2008, 163:84-99

38. Pedraza CE, Nikolcheva LG, Kaartinen MT, Barralet JE, McKee MD Osteopontin functions as an opsonin and facilitates phagocytosis by macrophages of hydroxyapatite-coated microspheres: implications for bone wound healing. Bone 2008, 43:708-716

39. Cowan JK: Anterograde and retrograde transneuronal degeneration in the central and peripheral nervous system. Edited by Nauta WJ Ebbesson OE. New York, Springer, 1970, pp. 217-249

40. Herrmann G, Stunitz H, Nitsch C: Composition of ibotenic acidinduced calcifications in rat substantia nigra. Brain Res 1998, 786:205-214

41. Zhao Y, Urganus AL, Spevak L, Shrestha S, Doty SB, Boskey AL, Pachman LM: Characterization of dystrophic calcification induced in mice by cardiotoxin. Calcif Tissue Int 2009, 85:267-275

42. Speer MY, Chien YC, Quan M, Yang HY, Vali H, McKee MD, Giachelli CM: Smooth muscle cells deficient in osteopontin have enhanced susceptibility to calcification in vitro. Cardiovasc Res 2005, 66:324-333 